\title{
FH Special Issue: Health related quality of life in individuals at high risk for familial hypercholesterolemia undergoing genetic cascade screening in Brazil
}

\begin{abstract}
Ana Cristina Souto ${ }^{1}$, Marcio H. Miname ${ }^{1}$, Julia Fukushima ${ }^{1}$, Cinthia E. Jannes ${ }^{1}$, Jose E. Krieger ${ }^{1}$, Martin Hagger ${ }^{2,3,4}$, Alexandre C. Pereira ${ }^{1}$, Raul D. Santos ${ }^{1,5}$.
\end{abstract}

1-Heart Institute (InCor) University of Sao Paulo Medical School Hospital, Sao Paulo, Brazil

2- Faculty of Sport and Health Sciences, University of Jyva skyla", Finland

3-School of Psychology, Curtin University, Australia

4- School of Applied Psychology, Griffith University, Australia

5-Hospital Israelita Albert Einstein, Sao Paulo, Brazil

Key words: familial hypercholesterolemia; cascade screening; lowering-lipid therapy; health related life quality; self-reporting; cardiovascular disease

Correspondence:

Raul D. Santos

Unidade Clínica de Lípides-InCor HCFMUSP

Av. Dr Eneas. C Aguiar 44, Bloco 2 Segundo Andar Sala 4

Cep: 05403-000, São Paulo, Brazil

E mail: rdsf@uol.com.br 


\begin{abstract}
Background: Familial hypercholesterolemia $(\mathrm{FH})$ is a genetic disorder associated with high risk of early major cardiovascular events (MACE) that can impact health related quality of life (HRQoL), however, this association is unclear. This study evaluated HRQoL in index cases (IC) and first-degree relatives (FDR) of individuals at high risk of $\mathrm{FH}$ submitted to genetic cascade screening.
\end{abstract}

Methods: Data collection was performed before awareness of molecular diagnosis results. Individuals were divided into four groups according to molecular diagnosis: IC with (IC+) and without (IC-) identified mutations ( $n=175$ and $n=93$, respectively), and their affected (FDR+, $n=231$ ) and non-affected (FDR-, $n=159)$ FDR. HRQoL measurements, mental (MCS) and physical component (PCS) scores, were carried out with SF-12 questionnaire. Associations were tested by generalized linear models.

Results: The mean age was $49 \pm 15$ years, $42.2 \%$ were men, MACE had occurred in $30.7 \%$. Overall both PCS and MCS did not differ between FH and non-FH individuals, however IC trended to have lower PCS independent of FH presence $(p=0.003)$. Lower PCS were associated with female sex $(p=0.018)$, lower education $(p<0.001)$, professional inactivity $(p=0.028)$, previous MACE occurrence $(p<0.001)$, hypertension $(p=0.016)$, depression $(p<0.001)$ and obesity $(p<0.001)$. Lower MCS were associated with female sex $(p=0.009)$, previous MACE occurrence $(p=0.034)$, depression $(p<0.001)$ and smoking $(p=0.009)$. Neither the presence of FH causing 
mutations nor pharmacological lipid lowering treatment were associated with HRQoL.

Conclusions: HRQoL is not reduced in both IC and FDR FH individuals in comparison with their non-affected counterparts. Previous MACE and comorbidities are associated with reduced HRQoL. 


\section{Introduction}

Familial hypercholesterolemia $(\mathrm{FH})$ is a life-long genetic health condition characterized by elevated LDL-cholesterol (LDL-C) concentrations and is associated with an elevated risk of early atherosclerotic cardiovascular disease (ASCVD) and death. There is consensus that if diagnosed early FH can be successfully managed by use of statins and lifestyle change, lessening related morbidity and mortality (1). FH is usually detected among individuals with premature coronary heart disease in tertiary care centers, and diagnostic precision necessitates the use of genetic testing due to the autosomal dominant nature of molecular defects causing this disease(2). The detection of an index case (IC) facilitates cascade screening of first degree relatives (FDR) and other family members in sequence(3).

Despite strategies for detection, treatment, and management of $\mathrm{FH}$, barriers such as low awareness of the disease and its risks among general population as well as problems related to lipid lowering treatment adherence, misconceptions about the illness and the side effects of medication specially statins, or affordability are particularly important gaps which contribute to heath disparities maintenance $(1,4,5)$.

In a wide comprehensive conceptual model of prevention and health promotion, psychosocial factors assessment help to identify possible barriers to diagnosis, and adequate adherence to a healthy lifestyle and pharmacological treatment (5). It is known that psychosocial factors are risk modifiers that add complexity to cardiovascular risk prevention efforts (6). 
One important issue for the management of $\mathrm{FH}$ individuals is how this disorder could affect health related quality of life (HRQoL). The influence of disease related aspects like the presence of a molecular defect, previous ASCVD manifestations, pharmacological treatment as well as psychosocial aspects on HRQoL of FH individuals is uncertain especially due to widespread underdiagnosis of the disease (4, 7-9). Genetic cascade screening opens up the possibility of comparing HRQoL in confirmed FH IC as well as in their affected and non-affected FDR. Previous studies from European countries suggest that HRQoL is not reduced in $\mathrm{FH}$ patients $(7,9-11)$. However, due to sociocultural, educational, and economic differences it is unclear if these findings apply to Brazil, a country with high social and educational inequalities and where $\mathrm{FH}$ is severely underdiagnosed.

Identifying variation in HRQoL in genetically-confirmed FH ICs, relative to their non-confirmed counterparts will provide important information relevant to the in-clinic management of $\mathrm{FH}$ patients, and the development of interventions to minimize negative psychological effects of the disease. Thus, the objective of this study was to evaluate self-estimated HRQoL and its associated factors in Brazilian individuals participating on a $\mathrm{FH}$ genetic cascade screening program.

\section{Methods}

Study population

This is a cross-sectional pre-specified analysis of prospectively collected data from individuals participating in the Hipercol Brasil FH genetic cascadescreening program performed at the Heart Institute (InCor) University of Sao Paulo 
Medical School Hospital in Sao Paulo, Brazil (12). This study was approved by the local ethics committee, it follows the declaration of Helsinki orientations and a written informed consent was obtained from all participants.

The inclusion criteria of the present study were age $\geq 18$ years old in individuals with a clinical suspicion of $\mathrm{FH}$ e.g. presenting or referring a previous $\mathrm{LDL}-\mathrm{C} \geq 5.4 \mathrm{mmol} / \mathrm{L}(\mathrm{IC})$ or be a FDR that was invited to participate in the cascade screening program between April 2011 and May 2014. In addition, it was required that individuals filled-in the Medical Outcomes Study 12-Item Short-Form Health Survey (SF-12) questionnaire at the initial screening visit.

Molecular diagnosis

$\mathrm{FH}$ causing mutations were determined on the $\mathrm{LDL}$ receptor $(L D L R)$, apolipoprotein $\mathrm{B}(A P O B)$ and protein convertase subtilisin kexin type 9 (PCSK9) genes as previously described(12). In addition to gene sequencing, Multiplex Ligation-dependent Probe Amplification (MLPA) was performed to detect copy number variants.

Data collection

Data were collected at the first session of genetic diagnosis process, during anamnesis interview without any previous knowledge of genetic test results. Participants completed a questionnaire including self-report measures of sociodemographic and psychosocial ASCVD risk modifiers including age, sex, educational level ( $>12$ and $\leq 12$ years of study), professional status (professionally active or inactive); previous major cardiovascular event (MACE) status (angina 
pectoris, myocardial infarction, heart failure, arrhythmias and myocardium

revascularization both surgical or percutaneous); risk factors for ASCVD

(hypertension, type 2 diabetes, obesity and smoking), awareness of $\mathrm{FH}$ aspects

(hyperlipidemia diagnosis, clinical suspicion of $\mathrm{FH}$, family history of early ASCVD,

family history of hypercholesterolemia), use of pharmacological lipid lowering

therapy; depression diagnosis, and $\mathrm{HRQ}$ oL status. $\mathrm{HRQ}$ oL was investigated using

the SF-12 questionnaire previously validated in Brazil $(13,14)$. The summary

scores, the Physical Summary Component (PSC) and Mental Summary

Component (MSC), representing the physical and mental summary measures. For

the estimation of worst and best quality of life, values below and above the 50 ( \pm

10) of PSC and MSC for the studied population were chosen as previously done

for $\mathrm{FH}$ individuals (7).

Statistical Analysis

Study participants were divided in four groups according results of the genetic tests: IC and their FDR with or without a confirmed FH-causing mutation respectively IC+, FDR+, IC- and FDR-. Analysis of variance (ANOVA) was used to compare HRQOL levels in IC and FDR groups followed up with Dunn's multiple comparisons test. Generalised linear models were used to evaluate the association of studied parameters, expressed as $\beta$ coefficients and their $95 \%$ confidence intervals $(\mathrm{Cl})$, with HRQoL measures (PCS and MCS scores as continuous dependent variables) in the whole study cohort. Models were adjusted for age (per 10-year intervals), sex, educational level, professional status, previous MACE occurrence, risk factors for ASCVD (hypertension, diabetes, obesity, smoking, type 
2 diabetes), depression, lipid lowering pharmacological treatment, awareness of $\mathrm{FH}$ aspects, and presence of an $\mathrm{FH}$ causing mutation. Coefficients with positive and negative values represent respectively associations with increased and reduced HRQoL. All analyses were performed using the SPSS v.17.0 software (Chicago, IL, USA) and $\mathrm{p}$ values were adjusted for multiple comparison by Bonferroni correction if necessary.

\section{Results}

From 1,030 individuals submitted to cascade screening, $658(63.8 \%)$ had complete data for the analysis and were included in the study. The mean age was $49 \pm 15$ years, $42.2 \%$ were men $(n=278), 54.3 \%(n=358)$ had $\leq 12$ years of study, $58.4 \%(n=384)$ were professionally active and the mean (SD) LDL-C was $5.09 \pm$ $2.07 \mathrm{mmol} / \mathrm{L}$. The HRQoL PCS and MCS were $47.4 \pm 9.6$ (median 50.0, percentiles 25-75 42.8 and 54.90), and 48.5 \pm 9.2 (median 50.9, percentiles 25-75 44.1 and 55.7) respectively. More than half of studied participants $(51 \%, n=204)$ had been referred or summoned for cascade screening from outside InCor's Lipid outpatient clinic. Overall, LDL receptor and $A P O B$ mutations were encountered in $49.2 \%$ ( $\mathrm{n}=324$ ) of individuals, no mutations in PCSK9 were found.

MACE had occurred in $30.7 \%(n=202)$ of the population. Lipid lowering pharmacological therapy, family history of hypercholesterolemia, familial history of early ASCVD, hyperlipidemia awareness and clinical suspicion of $\mathrm{FH}$ were reported by $81.2 \%, 70.6 \%, 60.8 \%, 75.7 \%$, and $20.2 \%$ of individuals respectively. The frequencies of referred depression, type 2 diabetes, smoking and hypertension were respectively $13.7 \%, 11.1 \%, 11.5 \%$, and $34.4 \%$. Supplementary table 1 shows 
that there were no significant differences on the studied characteristics between included and excluded individuals.

Table 1 shows sociodemographic, clinical and laboratory parameters of studied participants according to IC and FDR status. Overall, the frequency of MACE components (except for arrhythmias) was more common in $\mathrm{FH}$ individuals (both IC+ and FDR+) than in non-affected counterparts $(p<0.001) . I C+$ presented the highest MACE rates in comparison with other 3 groups. On the contrary, the lowest MACE frequencies were encountered in FDR- individuals. Significant differences were also observed on the frequency of hypertension that was highest in IC- $(p=0.019)$, hyperlipidemia that was greater in IC groups $(p<0.001)$, smoking that was highest in IC- $(p=0.045)$, use of pharmacological lipid-lowering therapy that was higher in both IC groups $(p<0.001)$, clinical suspicion of $\mathrm{FH}$ that was higher in $\mathrm{IC}+$ and FDR+ $(\mathrm{p}<0.001)$, and family history of hypercholesterolemia that was higher in IC- and FDR+ $(p<0.001)$. The highest and lowest LDL-C levels were encountered respectively in IC+ and IC- $(p<0.001)$. There were non-significant differences regarding sex, educational level, professional status, family history of early ASCVD, depression, obesity and type 2 diabetes frequencies among the groups.

Table 2 shows the PCS and MCS of studied groups. IC groups trended to have lower PCS in comparison with FDR $(p=0.003)$. However, the percentages of individuals with PCS below the worst quality of life threshold did not differ among groups ( $p=0.052)$. No differences were found in MCS $(p=0.338)$ and in the number of individuals with MCS below the worst quality of life threshold $(p=0.363)$ among 
the 4 studied groups. Furthermore, no significant differences were encountered in both HRQoL scores when participants were separated according to presence $(n=324)$ or not $(n=334)$ of an FH causing mutation: PCS (47.9 \pm 9.1 vs. $47.4 \pm 9.5$, $\mathrm{p}=0.663)$ and MCS $(49.6 \pm 8.5$ vs. $48.1 \pm 10.5, \mathrm{p}=0.219)$ respectively.

Table 3 shows the multivariate associations ( $\beta$ coefficients and 95\% Cl) of socio-demographic and clinical parameters as well as FH mutation status with PCS and MCS HRQoL components as dependent variables in the whole study cohort. Lower PCS were independently associated with female sex $(p=0.018)$, school level $\leq 12$ years $(p<0.001)$, professional inactivity status $(p=0.028)$, previous MACE occurrence $(p<0.001)$, hypertension $(p=0.007)$, depression $(p<0.001)$ and obesity $(p<0.001)$. On the other hand, previous clinical suspicion of $\mathrm{FH}$ was associated with higher PCS $(p=0.019)$. No associations were found however, with age, family history of either hypercholesterolemia or early ASCVD, and with use of lipid lowering pharmacological treatment.

Lower MCS were associated with female sex $(p<0.0001)$, previous MACE occurrence $(p=0.036)$, depression $(p<0.001)$ and smoking $(p=0.010)$. On the other hand, increasing age was associated with higher MCS $(p=0.012)$. There were no associations of $\mathrm{FH}$ awareness aspects with MCS. The presence of $\mathrm{FH}$ causing mutations were neither associated with PCS $(p=0.426)$ nor with MCS components $(p=0.062)$. Supplementary tables 2 and 3 show PCS and MCS according with presence or not of variables associated with HRQoL. 


\section{Discussion}

We examined HRQOL in individuals at high risk for $\mathrm{FH}$ from a genetic cascade screening program in Brazil. We found that HRQoL did not differ in patients with molecularly proven $\mathrm{FH}$, both $\mathrm{IC}$ and FDR, from that of non-affected counterparts. However, IC individuals probably due to their greater burden of previous ASCVD and co-morbidities, independently if $\mathrm{FH}$ diagnosis was confirmed, presented mean lower PCS HRQoL than their FDR. There were no differences in MCS among studied groups.

Previous observations in patients from Northern Europe $(9,11)$, not all with confirmed genetic FH diagnosis, and from cross-sectional Dutch (10) and Spanish studies with $100 \%$ molecular testing(7), suggest that $\mathrm{FH}$ per se is not associated with reduction in HRQoL. Our findings from a group of genetically proven $\mathrm{FH}$ individuals from a different sociocultural and educational background corroborates these outcomes.

In the current study, we could expand previous findings by comparing HRQoL according to IC and FDR status in an FH cascade screening program. Hitherto even in studies where FH was confirmed by molecular diagnosis, individuals had been separated as having $\mathrm{FH}$ or not independent of their status in the cascade screening program (i.e., IC or FDR with or without FH as confirmed by genetic testing) $(7,10)$. In the current study, PCS was significantly lower in IC participants than in FDR participants independent of FH presence. This was likely due to a greater burden of previous MACE and co-morbidities in these individuals. Reduced HRQoL in both FH and non-affected individuals was related to 
psychosocial factors like female sex, lower educational level, referred risk factors for ASCVD other than FH, like obesity, smoking and hypertension, depression and previous occurrence of MACE. These variables had been previously associated with lower HRQoL in European FH individuals in the studies of van Marle et al. and of Mata et al $(7,8)$. However, in contrast to the latter study in which previous MACE was associated with higher MCS, we found the opposite effects. According to Mata et al. (5) this could have been attributed to patient adaptation since these individuals had previously been submitted to a life-threatening situation. Differences in age, participants in this study were on average 10 years older than the ones from Spain, education and other sociocultural factors, as well as the greater prevalence of MACE in the Brazilian cohort (35\% vs. $14 \%$ in Brazilian and Spanish populations), may potentially explain these variations across samples and is an avenue for future research.

Importantly, the presence of an $\mathrm{FH}$-causing mutation, an accepted surrogate of elevated lifetime exposure to hypercholesterolemia and an independent marker of greater ASCVD risk (15), was not associated with reduced HRQoL. This finding is reinforced by the fact that participants submitted to cascade screening in this study were not aware of genetic test results when they were interviewed. Previous evidence however, suggests that awareness of $\mathrm{FH}$ genetic test result might influence on risk perception and management $(10,16)$, facts that could impact on MACE occurrence and consequently on HRQoL. However, considering the crosssectional nature of this study, the influence of knowledge of the test results on $\mathrm{HRQoL}$, rather than the $\mathrm{FH}$ mutation per se, needs to be evaluated prospectively. 
Roughly one in three and one in four of IC+ and FDR+ cases, respectively, reported an awareness of a clinical suspicion of $\mathrm{FH}$, and this was significantly higher than in individuals where mutations were not encountered. This pattern is in accord with the autosomal dominant trait of $\mathrm{FH}$ even considering that with exception of IC-, hyperlipidemia was referred by more than $87 \%$ of studied individuals of the other two groups. One interesting finding is that clinical suspicion of $\mathrm{FH}$, a genetic disease that runs within affected families and implicates in a greater risk of early ASCVD and mortality, was paradoxically associated with higher PCS. This deserves some considerations: (a) there is evidence that the presence of a genetic disease, even considering the physical consequences of that given disease, does not necessarily mean reduction in HRQoL and indeed may indicate the opposite (17); and (b) it seems reasonable to argue that most of the studied cohort would be expecting $\mathrm{FH}$ molecular confirmation since the majority had already a non-genetic diagnosis for hyperlipidemia, and the benefit of accessing or continuing consulting at an excellence centre like InCor's lipid outpatient clinic (roughly $50 \%$ of studied participants), could have influenced on their quality of life perception.

Lipid lowering pharmacological therapy, especially with statins, that were used by most IC and FDR+ in our study, reduces the risk of MACE in FH individuals and therefore has the potential to improve HRQoL as seen in the general population $(18,19)$. For example, a cross-sectional Danish study of molecularly-proven $\mathrm{FH}$ individuals, albeit in a small sample without previous occurrence of MACE, suggested that HRQoL could be reduced in individuals 
where LDL-C was inadequately controlled(20). On the other hand, there is misconception on health literacy regarding risks of statin treatment (21), that is usually referred as cause of muscular symptoms among other possible adverse events(22). Therefore, statin use might negatively influence individuals' HRQoL perceptions, especially in those that are asymptomatic with no previous occurrence of MACE as is frequently encountered in FDR participating in cascade screening. One important finding of our study is that pharmacological treatment was not associated with reduced HRQoL. Whether the high prevalence of MACE in the population and the awareness of possible benefits of statin treatment for these individuals influences on these findings remains to be determined. At any rate, these findings may help convince $\mathrm{FH}$ individuals not only to start but also adhere to the necessary lifelong pharmacological therapy.

Our study confirms and expands previous findings from European studies to individuals in a cascade screening program in Brazil that differ from the former in sociocultural, educational, and medical aspects. The common finding that comorbidities, especially previous MACE, are associated with HRQoL not only in FH but in their non-affected counterparts needs to be emphasized. We speculate that reduction in HRQoL, particularly in patients with MACE, is mainly associated with preventive management failure. The analysis suggests that despite knowledge of a genetically-verified $\mathrm{FH}$ diagnosis, $\mathrm{CV}$ prevention and $\mathrm{FH}$ management is usually initialized too late. We hope this is changing as the genetic $\mathrm{FH}$ cascade screening in Brazil reaches people from across this vast, multicultural nation. Early 
diagnosis leading to early treatment might overall lead to control of co-morbidities and MACE prevention contributing to improved HRQoL over time.

The current study has some limitations, its cross-sectional design allows us to identify associations rather than causality. Long-term prospective studies to confirm the associations encountered in this and on previous observations need to be performed. Information about co-morbidities and MACE was ascertained by individuals answering a questionnaire and are potentially subject to error variance due to poor recall.

In conclusion HRQoL is not reduced in both IC and FDR cases in Brazilian $\mathrm{FH}$ individuals submitted to a genetic cascade screening program in comparison with their non-affected counterparts. IC independent of FH status had reduced physical HRQoL in comparison with FDR, likely due to a greater prevalence of MACE and other co-morbidities. In addition, neither the presence of a FH causing mutation nor pharmacological lipid lowering therapy were associated with HRQoL. Early diagnosis and treatment of risk factors and co-morbidities in individuals submitted to cascade screening may reduce the risk of MACE and might improve HRQoL.

\footnotetext{
Author contributions: RDS, AP, ACS, CEJ and JEK have designed the study; JF, ACS, MHM have analyzed the data; ACS, RDS, MH and MHM have written the paper. All authors have reviewed and approved the manuscript.
} 
Conflicts of interest declaration:

RDS has received honoraria for consulting, speaker and or research activities

from: Akcea, Amgen, Astra Zeneca, Biolab, Kowa, Pfizer, Merck, Novo Nordisk

and Sanofi/Regeneron. The other authors have no conflicts to declare.

References

1. Gidding SS, Ann Champagne M, de Ferranti SD, Defesche J, Ito MK, Knowles JW, et al. The Agenda for Familial Hypercholesterolemia: A Scientific Statement From the American Heart Association. Circulation. 2015;132:2167-92.

2. Watts GF, Pang J. The evolving model of care for familial hypercholesterolaemia. Eur J Prev Cardiol. 2017;24(16):1729-32.

3. Santos RD, Frauches TS, Chacra AP. Cascade Screening in Familial

Hypercholesterolemia: Advancing Forward. J Atheroscler Thromb. 2015;22(9):869-80.

4. Nordestgaard BG, Chapman MJ, Humphries SE, Ginsberg HN, Masana L,

Descamps OS, et al. Familial hypercholesterolaemia is underdiagnosed and undertreated in

the general population: guidance for clinicians to prevent coronary heart disease: consensus statement of the European Atherosclerosis Society. Eur Heart J. 2013;34(45):3478-90a.

5. Hagger MS, Hardcastle SJ, Hingley C, Strickland E, Pang J, Watts GF. Predicting

Self-Management Behaviors in Familial Hypercholesterolemia Using an Integrated

Theoretical Model: the Impact of Beliefs About Illnesses and Beliefs About Behaviors. Int J Behav Med. 2016;23(3):282-94.

6. Authors/Task Force M, Piepoli MF, Hoes AW, Agewall S, Albus C, Brotons C, et al. 2016 European Guidelines on cardiovascular disease prevention in clinical practice: The Sixth Joint Task Force of the European Society of Cardiology and Other Societies on Cardiovascular Disease Prevention in Clinical Practice (constituted by representatives of 10 societies and by invited experts) Developed with the special contribution of the European Association for Cardiovascular Prevention \& Rehabilitation (EACPR). Atherosclerosis. 2016;252:207-74.

7. Mata N, Alonso R, Banegas JR, Zambon D, Brea A, Mata P. Quality of life in a cohort of familial hypercholesterolemia patients from the south of Europe. Eur J Public Health. 2014;24(2):221-5.

8. van Maarle MC, Stouthard ME, Bonsel GJ. Quality of life in a family based genetic cascade screening programme for familial hypercholesterolaemia: a longitudinal study among participants. J Med Genet. 2003;40(1):e3.

9. Hollman G, Gullberg M, Ek AC, Eriksson M, Olsson AG. Quality of life in patients with familial hypercholesterolaemia. Journal of internal medicine. 2002;251(4):331-7.

10. van Maarle MC, Stouthard ME, Bonsel GJ. Risk perception of participants in a family-based genetic screening program on familial hypercholesterolemia. Am J Med Genet A. 2003;116A(2):136-43. 
11. Hyttinen L, Kekalainen P, Vuorio AF, Sintonen H, Strandberg TE. Health-related quality of life in elderly patients with familial hypercholesterolemia. Int J Technol Assess Health Care. 2008;24(2):228-34.

12. Jannes CE, Santos RD, de Souza Silva PR, Turolla L, Gagliardi AC, Marsiglia JD, et al. Familial hypercholesterolemia in Brazil: cascade screening program, clinical and genetic aspects. Atherosclerosis. 2015;238(1):101-7.

13. de Abreu MM, Walker DR, Sesso RC, Ferraz MB. Health-related quality of life of patients recieving hemodialysis and peritoneal dialysis in Sao Paulo, Brazil: a longitudinal study. Value Health. 2011;14(5 Suppl 1):S119-21.

14. Silveira MF, Almeida JC, Freire RS, Haikal DS, Martins AE. [Psychometric properties of the quality of life assessment instrument: 12-item health survey (SF-12)]. Cien Saude Colet. 2013;18(7):1923-31.

15. Khera AV, Won HH, Peloso GM, Lawson KS, Bartz TM, Deng X, et al. Diagnostic Yield and Clinical Utility of Sequencing Familial Hypercholesterolemia Genes in Patients With Severe Hypercholesterolemia. J Am Coll Cardiol. 2016;67(22):2578-89.

16. Senior V, Marteau TM, Peters TJ. Will genetic testing for predisposition for disease result in fatalism? A qualitative study of parents responses to neonatal screening for familial hypercholesterolaemia. Soc Sci Med. 1999;48(12):1857-60.

17. Cohen JS, Biesecker BB. Quality of life in rare genetic conditions: a systematic review of the literature. Am J Med Genet A. 2010;152A(5):1136-56.

18. Besseling J, Hovingh GK, Huijgen R, Kastelein JJ, Hutten BA. Statins in Familial Hypercholesterolemia: Consequences for Coronary Artery Disease and All-Cause Mortality. J Am Coll Cardiol. 2016;68(3):252-60.

19. Taylor F, Huffman MD, Macedo AF, Moore TH, Burke M, Davey Smith G, et al. Statins for the primary prevention of cardiovascular disease. Cochrane Database Syst Rev. 2013(1):CD004816.

20. Mortensen GL, Madsen IB, Kruse C, Bundgaard H. Familial hypercholesterolaemia reduces the quality of life of patients not reaching treatment targets. Dan Med J. 2016;63(5).

21. Santos RD. Better health literacy can make the difference when control of risk factors for cardiovascular disease and quality of life are concerned. Eur J Prev Cardiol. 2017;24(17):1878-9.

22. Gupta A, Thompson D, Whitehouse A, Collier T, Dahlof B, Poulter N, et al. Adverse events associated with unblinded, but not with blinded, statin therapy in the Anglo-Scandinavian Cardiac Outcomes Trial-Lipid-Lowering Arm (ASCOT-LLA): a randomised double-blind placebo-controlled trial and its non-randomised non-blind extension phase. Lancet. 2017;389(10088):2473-81. 
Table 1 Sociodemographic and clinical aspects in affected (mutation +) and non- affected (mutation -) Familial Hypercholesterolemia index cases (IC) and first-degree relatives (FDR).

\begin{tabular}{llllll}
\hline & \multicolumn{5}{c}{ Cohort $(\mathbf{n}=658)$} \\
Variables & IC+ & IC- & FDR+ & FDR- & p value \\
& $(n=93)$ & $(n=175)$ & $(n=231)$ & $(n=159)$ & \\
\hline
\end{tabular}

\section{Demographics}

$\begin{array}{llllll}\text { Age (years) } & 50 \pm 14 & 52 \pm 12.3^{\mathrm{d}, \mathrm{e}} & 47 \pm 15.9^{\mathrm{d}} & 48 \pm 15.5^{\mathrm{e}} & .010 \\ \text { Male sex } & 41(44.1 \%) & 64(36.8 \%) & 102(44.2 \%) & 71(44.7 \%) & .368 \\ \leq 12 \text { years of study } & 49(52.7 \%) & 92(52.6 \%) & 134(54 \%) & 83(52.2 \%) & .601 \\ \text { Professionally active } & 56(60.2 \%) & 107(61.1 \%) & 149(64.5 \%) & 112(70.4 \%) & .256\end{array}$

\section{Clinical aspects}

$\begin{array}{llllll}\text { MACE } & 45(48.4 \%)^{\mathrm{a}, \mathrm{b}, \mathrm{c}} & 63(36 \%)^{\mathrm{a}, \mathrm{e}} & 69(29.9 \%)^{\mathrm{b}, \mathrm{f}} & 25(15.7 \%)^{\mathrm{c}, \mathrm{e}, \mathrm{f}} & <.001 \\ \text { Heart failure } & 13(14 \%)^{\mathrm{b}, \mathrm{c}} & 17(9.7 \%)^{\mathrm{e}} & 12(5.2 \%)^{\mathrm{b}} & 4(2.5 \%)^{\mathrm{c}, \mathrm{e}} & .002 \\ \text { Angina pectoris } & 22(23.7 \%)^{\mathrm{b}, \mathrm{c}} & 27(15.4 \%)^{\mathrm{e}} & 27(11.7 \%)^{\mathrm{b}} & 11(6.9 \%)^{\mathrm{c}, \mathrm{e}} & .001\end{array}$




$\begin{array}{llllll}\text { Myocardial infarction } & 21(22.6 \%)^{\mathrm{b}, \mathrm{c}} & 31(17.7 \%)^{\mathrm{e}} & 26(11.3 \%)^{\mathrm{b}, \mathrm{f}} & 5(3.1 \%)^{\mathrm{c}, \mathrm{e}, \mathrm{f}} & <.001 \\ \text { Arrhythmias } & 14(15 \%) & 21(12 \%) & 25(10.8 \%) & 11(6.9 \%) & .213 \\ \text { Myocardium } & 27(29 \%)^{\mathrm{a}, \mathrm{b}, \mathrm{c}} & 21(12 \%)^{\mathrm{a}, \mathrm{e}} & 37(16 \%)^{\mathrm{b}, \mathrm{f}} & 3(1.89 \%)^{\mathrm{c}, \mathrm{e}, \mathrm{f}} & <.001 \\ \text { revascularization } & & & & \\ \text { Hypertension } & 29(31.2 \%)^{\mathrm{a}} & 76(43.2 \%)^{\mathrm{a}, \mathrm{d}, \mathrm{e}} & 78(33.8 \%)^{\mathrm{d}} & 44(27.7 \%)^{\mathrm{e}} & .019 \\ \text { Type 2 Diabetes } & 9(9.7 \%) & 19(10.9 \%) & 28(12.1 \%) & 17(10.7 \%) & .925 \\ \text { Obesity } & 11(11.8 \%) & 27(15.4 \%) & 24(10.4 \%) & 24(15.1 \%) & .390 \\ \text { Smoking } & 8(8.6 \%)^{\mathrm{a}} & 30(17.1 \%)^{\mathrm{a}, \mathrm{d}, \mathrm{e}} & 25(10.8 \%)^{\mathrm{d}} & 13(8.2 \%)^{\mathrm{e}} & .045 \\ \text { Depression } & 16(17.2 \%) & 35(20 \%)^{\mathrm{d}, \mathrm{e}} & 24(10.4 \%)^{\mathrm{d}} & 15(9.4 \%)^{\mathrm{e}} & .010 \\ \text { Pharmacological } & 86(92.5 \%)^{\mathrm{b}, \mathrm{c}} & 151(86.3 \%)^{\mathrm{d}, \mathrm{e}} & 175(75.8 \%)^{\mathrm{b}, \mathrm{d}, \mathrm{f}} & 53(33.3 \%)^{\mathrm{c}, \mathrm{e}, \mathrm{f}} & <.001 \\ \text { therapy } & & & & \\ \text { Baseline LDL-C } & 6.85 \pm 2.6^{\mathrm{a}, \mathrm{b}, \mathrm{c}} & 5.27 \pm 1.50^{\mathrm{a}, \mathrm{e}} & 5.42 \pm 1.83^{\mathrm{b}, \mathrm{f}} & 3.30 \pm 1.00^{\mathrm{c}, \mathrm{e}, \mathrm{f}} & <.001 \\ \text { (mmol/L) } & & & & \\ \text { Awareness of FH aspects } & 93(100 \%)^{\mathrm{b}, \mathrm{c}} & 172(98.3 \%)^{\mathrm{d}, \mathrm{e}} & 203(87.9 \%)^{\mathrm{b}, \mathrm{d}, \mathrm{f}} & 66(41.5 \%)^{\mathrm{c}, \mathrm{e}, \mathrm{f}} & <.001 \\ \text { Hyperlipidemia } & 34(36.6 \%)^{\mathrm{a}, \mathrm{c}} & 22(12.5 \%)^{\mathrm{a}, \mathrm{d}} & 64(27.7 \%)^{\mathrm{d}, \mathrm{f}} & 13(8.2 \%)^{\mathrm{c}, \mathrm{f}} & <.001 \\ \text { FH clinical suspicion } & & & & \end{array}$


ASCVD

Family history of

hypercholesterolemia

Legend for table 1: Categorical data $\mathrm{n}(\%)$; continuous variables mean \pm standard deviation

Similar alphabet letters mean $p$ values $<0.05$ on ANOVA post tests

a Comparison between IC+ and IC-

${ }^{\mathrm{b}}$ Comparison between IC+ and FDR+

${ }^{\mathrm{C}}$ Comparison between $\mathrm{IC}+$ and FDR-

${ }^{\mathrm{d}}$ Comparison between IC- and FDR+

${ }^{e}$ Comparison between IC- and FDR-

${ }^{f}$ Comparison between FDR+ and FDR- 
Table 2. Health-related quality of life (HRQoL) physical (PCS) and mental (MCS) scores in affected (mutation + ) and non- affected (mutation -) Familial Hypercholesterolemia index cases (IC) and first-degree relatives (FDR).

\begin{tabular}{|c|c|c|c|c|c|}
\hline & \multicolumn{4}{|c|}{ Cohort $(n=658)$} & \multirow[b]{2}{*}{$p$ value } \\
\hline & $\begin{array}{l}\text { IC+ } \\
(n=93)\end{array}$ & $\begin{array}{l}\text { IC- } \\
(n=175)\end{array}$ & $\begin{array}{c}\text { FDR + } \\
(n=231)\end{array}$ & $\begin{array}{l}\text { FDR - } \\
(n=159)\end{array}$ & \\
\hline \multicolumn{6}{|c|}{ Physical Component Score (PCS) } \\
\hline Mean (SD) & $47 \pm 9.5^{\mathrm{a}}$ & $45.6 \pm 10.1^{\text {b.c }}$ & $48.3 \pm 8.8^{b}$ & $49.4 \pm 8.4^{a . c}$ & .003 \\
\hline Range (min-max) & $(19.1-62.7)$ & $(16.7-62.1)$ & $(17.2-63.9)$ & $(17.5-63.8)$ & \\
\hline \multicolumn{6}{|l|}{$\%(n)$ with scores $\leq 50$} \\
\hline (worst quality of life) & $51.6 \%(48)$ & $56.6 \%(99)$ & $50.6 \%(117)$ & $41.5 \%(66)$ & .052 \\
\hline \multicolumn{6}{|c|}{ Mental Component Score (MCS) } \\
\hline Mean (SD) & $50.2 \pm 7.7$ & $47.4 \pm 10.6$ & $49.4 \pm 8.7$ & $48.8 \pm 10.2$ & .338 \\
\hline Range (min-max) & $(21.1-64.5)$ & $(21.2-68.5)$ & $(22.4-66.9)$ & $(11.7-67.2)$ & \\
\hline \multirow{2}{*}{\multicolumn{6}{|c|}{$\begin{array}{l}\%(\mathrm{n}) \text { with scores } \leq 50 \\
\text { (worst quality of life) }\end{array}$}} \\
\hline & & & & & \\
\hline & $43 . \%(40)$ & $44.6 \%(78)$ & $45 \%(104)$ & $43.4 \%(69)$ & .363 \\
\hline
\end{tabular}


Legend for table 2: Similar alphabet letters mean $p$ values $<0.05$ on ANOVA post tests 
Table 3. Multivariate associations with Health-Related Quality of Life (HRQoL): Physical (PCS) and Mental (MCS) components in the whole study cohort.

\begin{tabular}{|c|c|c|c|c|c|c|c|c|}
\hline \multirow{3}{*}{ Variables } & \multicolumn{8}{|c|}{ Cohort $(n=658)$} \\
\hline & \multicolumn{4}{|c|}{ PCS } & \multicolumn{4}{|c|}{ MCS } \\
\hline & \multirow[t]{2}{*}{$\beta$} & \multicolumn{2}{|c|}{$(95 \% \mathrm{Cl})$} & \multirow[t]{2}{*}{$\mathbf{P}$} & \multirow[t]{2}{*}{$\beta$} & \multicolumn{2}{|c|}{$(95 \% \mathrm{Cl})$} & \multirow[t]{2}{*}{$\mathbf{P}$} \\
\hline \multicolumn{5}{|l|}{ Demographics } & & & & \\
\hline Age (10 year & -.071 & -.438 & .297 & .706 & .508 & .112 & .904 & .012 \\
\hline \multicolumn{9}{|l|}{ intervals) } \\
\hline Female sex & -1.629 & -2.972 & -.285 & .018 & -3.290 & -4.741 & -1.838 & $<.001$ \\
\hline $\begin{array}{l}\text { School level } \\
\text { ( } \leq 12 \text { years) }\end{array}$ & -3.183 & -4.541 & -1.825 & $<.001$ & -.809 & -2.276 & .659 & .280 \\
\hline Professional inactivity & -2.060 & -3.595 & -.525 & .009 & 1.164 & -.494 & 2.823 & .168 \\
\hline \multicolumn{9}{|l|}{ Clinical aspects } \\
\hline MACE & -3.782 & -5.269 & -2.295 & $<.001$ & -1.717 & -3.324 & -.111 & .036 \\
\hline Hypertension & -2.105 & -3.624 & -.586 & .007 & 1.322 & -.319 & 2.963 & .114 \\
\hline
\end{tabular}




\begin{tabular}{|c|c|c|c|c|c|c|c|c|}
\hline Type 2 Diabetes & -.853 & -2.960 & 1.253 & .427 & -1.468 & -3.744 & .808 & .206 \\
\hline Depression & -3.552 & -5.437 & -1.667 & $<.001$ & -8.821 & -10.857 & -6.784 & $<.001$ \\
\hline Obesity & -3.399 & -5.288 & -1.511 & $<.001$ & -1.296 & -3.336 & .745 & .213 \\
\hline Smoking & -1.163 & -3.138 & .813 & .248 & -2.804 & -4.938 & -.669 & .010 \\
\hline $\begin{array}{l}\text { Pharmacological } \\
\text { therapy }\end{array}$ & -.476 & -2.408 & 1.456 & .628 & 1.703 & -.384 & 3.791 & .110 \\
\hline \multicolumn{9}{|c|}{ Awareness of FH aspects } \\
\hline Hyperlipidemia & -1.257 & -3.439 & .925 & .258 & -2.067 & -4.424 & .291 & .086 \\
\hline FH clinical suspicion & 1.921 & .322 & 3.519 & .019 & .370 & -1.357 & 2.097 & .674 \\
\hline $\begin{array}{l}\text { Family history of } \\
\text { early ASCVD }\end{array}$ & .667 & -.668 & 2.001 & .327 & -.186 & -1.628 & 1.255 & .800 \\
\hline $\begin{array}{l}\text { Family history of } \\
\text { hypercholesterolemia }\end{array}$ & .191 & -1.320 & 1.701 & .804 & .706 & -.926 & 2.339 & .396 \\
\hline \multicolumn{9}{|c|}{ Groups defined by molecular diagnosis } \\
\hline $\begin{array}{l}\text { FH causing mutation } \\
\text { presence }\end{array}$ & .548 & -.802 & 1.897 & .426 & 1.531 & .077 & 2.985 & .062 \\
\hline
\end{tabular}

Negative signs indicate reduction in HRQoL 